Aus der Klinik für Hautkrankheiten der Universität Strassburg i. . .

(Direktor: Prof. Dr. A. Wolff.)

\title{
Ein Beitrag zur Kenntnis ungewöhnlicher Keratosisformen.
}

\author{
Von \\ Dr. med. C. Gutmann,
}

I. Assistenzarzt.

(Hiezu Taf. IX.)

Auf dem weiten Gebiete der Keratosen bzw. Hyperkeratosen herrschen, wie schon ein flüchtiger Blick in die betreffenden Kapitel der dermatologischen Lehr- und Handbücher und speziell in die Einzelpublikationen über diesen Gegenstand zeigt, die größten Meinungsverschiedenheiten unter den Autoren. Ganz besonders treten diese Differenzen bei den ungewöhnlichen, nicht ganz in den Rahmen uns geläufiger und sicher feststehender Krankheitsbilder hinein passenden Formen von Keratosis zu Tage; dabei macht sich dann die große Neigung der Autoren, oft auf Grund einer einzigen und vielfach nur unbedeutend von bereits Bekanntem abweichenden Beobachtung ein neues Krankheitsbild zu schaffen und diesem natürlich auch einen neuen Namen zu verleihen, in höchst störender das Studium dieser Dinge nicht gerade erleichternder und eine Verständigung darüber keineswegs fördernder Weise geltend. Zum Beweise für die Richtigkeit des eben Gesagten möchte ich hier nur kurz an die in Frankreich geschaffene Krankheitsgruppe der acne sebacée cornée erinnern (Cazenave, Hardy, Guibont). Leloir und Vidal streichen von dieser $\mathrm{Be}-$ zeichnung das Beiwort sebacée, während Besnier von Angiofolliculite keratosique simple spricht. Nun ist aber Brooke 
der Meinung, daß diese Fälle aile in dieselbe Kategorie gehörfen wie seine Keratosis follicularis contagiosa, ebenso wie die Ichthyosis sebacea cornea Wilson, die Ichthyosis follicularis Lesser, die Keratosis follicularis Morrow und vielleicht auch einige der unter dem Namen Lichen spinulosus beschriebenen Krankheitsfälle. Brooke steht also, wenigstens was die acné cornée betrifft, durchaus auf demselben Standpunkt, den später To u to on vertritt, der mindestens die reinen Fälle von acné cornée zu den Keratosen gerechnet wissen will. Iob teile diese Ansicht Toutons durchaus, muß es aber andererseits unentschieden lassen, ob die weitere Meinung Tout ons, daß es sich in solchen Fällen um eine follikuläre Ichthyosis handele, zu Recht besteht. Auch die Entscheidung darüber, $a b$ diese reinen Formen ron acné cornée in verwandtschaftlichen Beziehungen zur Darierschen Psorospermose stehen, mu weiteren Untersuchungen vorbehalten bleiben. Dasselbe gilt für $\mathrm{N}$ e is s e r s Keratosis follicularis punctata et striata, wie auch Neisser selbst betont, und ferner für Hans Hebras Hyperkeratosis striata et follicularis, während beispielsweise die Identität der beiden durch White mitgeteilten Fälle von Keratosis follicularis mit der Darie rschen Krankheit vom Autor selbst sichergestellt ist. Auf die Fälle von Keratosis follicularis, über die Blaschko, Saalfeld u. a. zum Teil nur sehr kurz berichteten, ganz speziell aber auf die jüngst ausführlich mitgeteilte $\breve{S}$ a $\mathrm{m}$ b e r g e r sche Beobachtung, welcher der Autor die Bezeichnung Keratosis pseudofollicularis beilegt, wird weiter unten noch einzugehen sein.

Aus diesen Ausführungen wird das eine zur Genüge hervor gehen, daß unsere Anschauungen namentlich auf dem Gebiete ungewöhnlicher Formen von Keratosis noch keineswegs geklärt sind, und es erscheint mir daher jeder noch so bescheidene Beitrag zu diesem Kapitel der Dermatologie berechtigt, zumal wenn man, wie in dem Falle unserer Beobachtung, über den ich nunmehr berichten möchte, in die Lage versetzt ist, relativ reichliches Material zur mikroskopischen Untersuchung zu erbalten.

Am 11./VIII. 1905. erschien die 54jährige Witwe M. Sch. zum ersten Male in unserer poliklinischen Sprechstunde. Sie gab auf Befragen an, stets eine glatte Haut und eine gewöhnliche, nur im Sommer an den offen getragenen Stellen gebräunte Hautfarbe besessen zu haben. Vor 
einer Reihe von Jahren hat Pat. eine Kopfoperation durchgemacht, von der eine lineäre, mehrere centimeter lange Narbe in der Mittellinie des Halses herrührt. Die jetzige Erkrankung soll im Februar 1905 mit Jucken an den Vorderarmen und auf dem Kopfe, bald darauf auch im Gesicht und Nacken begonnen haben, ohne daß zunächst an der Haut etwas auffälliges zu bemerken gewesen wäre. Erst nach und nach sollen sich dann die Veränderungen entwickelt haben, wegen deren die Patientin unsere Hilfe in Anspruch nimmt. - Status: Bei der außerordentlich woblgenährten, vortrefflich aussehenden Frau findet sich eine Erkrankung der Haut, an folgenden Körperteilen lokalisiert: an der Streckseite der Mittel- und Grundphalangen sämtlicher Finger, an beiden Handrücken, an der Streckseite beider Vorderarme, aber auch weit übergreifend auf deren Beugefäche. Ferner sind befallen der Nacken, das Gesicht mit Ausnahme der Gegend um den Mund, die Obren und der behaarte Kopf. Die Ausbreitung der Affektion ist eine durchaus gleichmäßige, symmetrische.

An den Fingern, auf den Handrücken, besonders zahlrcich jedoch auf den Vorderarmen sieht man kleinste, papulöse, häufig konisch zugespitzte Effloreszenzen von höchstens Stecknadelkopfgröße, die außerordentlich hart sind und in ihrer Mitte sehr oft ein schwarzes Pünktchen oder auch ein abgebrochenes Haar erkennen lassen. Die Knötchen sind diffus verteilt, und lassen keine Neigung zu Gruppenbildung erkennen. Die dazwischen liegende Hant erscheint deutlicher gefeldert wie unter gewöhnlichen Verhältnissen und diffus braun pigmentiert, weniger jedoch auf der Benge- wie auf der Streckseite. Sonstige Veränderungen z. B. Schuppung, Rötung usw. lassen sich nicht konstatieren. Gleitet man mit dem Finger über die Haut hin, so hat man die Empfindung, als fahre man über ein Reibeisen.

Ganz auffallend pigmentiert erscheint das Gesicht. (Pat. gibt an, noch niemals während ihres Lebens in den Sommermonaten auch nur annähernd eine solche Gesichtsfarbe gehabt zu haben.) Besonders intensiv verfärbt, ausgesprochen bronzefarben, präsentiert sich die Haut der Stirn, um die Augen herum und namentlich in der Schläfen- und Jochbeingegend. Die übrigen Teile des Gesichtes, der Nacken und Hals weisen eine mehr oder minder diffuse und tiefe Braun- resp. Rotbraunfärbung auf. Am wenigsten pigmentiert ist die Partie um den Mund; keine Pigmentationen laasen sich auf der Zungen-, Mund- und Rachenschleimhaut erkennen. Im Bereiche dieser Pigmentierung treten nun die sämtlichen Follikel als kleine, schwarze Pünktchen und Fleckchen hervor, wie das besonders schön an der Innenund Außenfläche der Ohrmuscheln zu sehen ist, oder aber man sieht kleinste, sehr derbe, weißlich oder auch schmutzig-grau verfärbte, häufig zugespitzte Papeln. Anch hier hat der darüber streichende Finger das Gefühl, als gleite er über eine Küchenreibe.

Auch die Follikel auf dem behaarten Kopfe zeigen sich als schwarze, kaum erhabene Punkte, und zwar ist der Prozeß in der Hauptsache auf dem Vorderkopf entwickelt. Die Seitenteile des Kopfes und vor allem der Scheitel sind mit dicken, grobblättrigen, vollkommen trockenen 
Schuppenmassen bedeckt. Die Haare stehen an sich zwar nicht sehr dicht, doch ist auch nirgends ein deutlicher Haarausfall zu konstatieren, und die Haare selbst bieten keine dem Auge wahrnehmbaren Veränderungen dar.

Soweit die Haut in der eben besehriebenen Weise erkrankt ist, erscheint sie auffallend trocken, und die Pat. erklärt auf das bestimmteste, im Gegensatz zu früheren Zeiten in diesem Sommer auf ihrem Gesicht niemals einen Schweißtropfen bemerkt zu haben.

Die Haut des übrigen Körpers weist außer einem ganz geringen Grade von Keratosis pilaris an der Streckseite der Oberarme und Oberschenkel keine Veränderungen auf, abgesehen davon, dah im Bereiche der oberen Hälfte der Beugeseite der Untersahenkel ziemlich zahlreiche Follikel als hornige, konische Effloreszenzen wahrzunehmen sind. Pigmentation feblt hier vollständig, ebenso Juckreiz, wie Pat. ganz bestimmt versichert. Wie lange diese letzteren Gebilde bestehen, vermag Pat. nicht zu sagen.

Es liegen hier also im wesentlichen zwei Prozesse vor, nämlich eine circumscripte, klinisch fast ausschließlich auf die Follikel beschränkte Hyperkeratose, und ferner eine innerhalb der Grenzen dieser Veränderungen sich haltende ungewöhnliche Pigmentierung. Wir mußten uns demgemäß folgende Fragen vorlegen: Bestehen diese beiden krankhaften Prozesse neben einander als Symptome zweier verschiedener Krankbeiten oder gehören beide einem Krankheitsbilde an? Und ferner wenn letzteres der Fall, um welche Krankheit handelt es sich hier?

Von Allgemeinerkrankungen, die mit Pigmentablagerung in der Haut einher gehen, waren vor allem zu berücksichtigen: die Arsenintoxikation, der Morbus Addisonii und der Bronzediabetes. Die erstere, bei der ja auch Hyperizeratosen sich entwickeln können, konnte ohne weiteres ausgeschlossen werden, da die Pat. niemals Arsen gesehen und noch viel weniger gebraucht hat. Auch der Morbus Addisonii mußte in Anbetracht des vortrefflichen Allgemeinzustandes und der körperlichen Leistungsfähigkeit, bei dem Fehlen von Pigmentflecken an den Schleimbäuten, bei dem Fehlen irgendwelcher Störungen von seiten des Zirkulationsapparates, des Digestionstraktus und des Nervensystems fallen gelassen werden. Und was den Diabète bronzé betrifft, so konnte auch dieser abgelehnt werden, weil, abgesehen von dem ausgezeichneten Allgemeinbefinden, die wichtigsten objektiven Symgtome desselben, die hypertrophische Lebercirrhose und die Ausscheidung von Zucker durch den Harn trotz wiederholter Untersuchung nicht konstatiert werden konnten. 
Endlich mußte auch an die Möglichkeit eines Zusammenhanges der hier vorliegenden Erscheinungen mit der vor Jahren ausgeführten Kropfoperation gedacht werden. Indes auch diesen Zusammenhang möchte ich mit Bestimmtheit ablehnen. Zunächst wissen wir ja nicht einmal, ob die Schilddrüse seinerzeit vollständig oder wieviel von ihr entfernt worden ist. Aber abgesehen davon sehen wir auch nicht das mindeste, was als Ausfallserscheinungen infolge fehlender Schilddrüsenfunktion gedeutet werden könnte, und vor allem finde ich in der hervorragenden, erschöpfenden Monographie von Eiselsbergs über die Krankheiten der. Schilddrüse auch nicht den mindesten Hinweis darauf, daß im Anschluß an partelle oder totale Kropfexstirpationen Erscheinungen der Haut, wie sie unsere Pat. darbietet, bisher beobachtet wären.

Wir müssen also für den vorliegenden Fall die Abhängigkeit der abnormen Hautpigmentierung von einer Allgemeinerkrankung des Organismus ablehnen. Unsere Auffassung des Falles geht vielmehr dahin, daß die Pigmentablagerung und die Hyperkeratose zu einem Krankheitsbilde gehören. Diese Anschauung wird zunächst gestützt durch die bestimmte Angabe der Pat., daß mit dem Auftreten der Hyperkeratose sich auch die Verfärbung der Haut eingestellt habe. Beweisend für diesen Zusammenhang scheint mir aber vor allem die Tatsache zu sein, daß Hyperkeratose und Pigmentierung in ihrer Ausdehnung absolut genau die gleichen Grenzen inne halten, und daß nirgends auf der Haut eine abgesprengte Pigmentinsel oder Keratosisplaque aufzufinden ist. Endlich sind ja auch Erkrankungen der Haut, zu deren Symptomen unter anderen hyperkeratotische Prozesse und abnorme Pigmentablagerungen gehören, hinlänglich bekannt.

Lassen wir nunmehr die Krankheiten der Haut, unter deren Erscheinungen diese beiden Prozesse eine mehr oder minder vorherrschende Rolle spielen, kurz Revue passieren. Es kommen da in Betracht: die Ichthyosis, der Lichen bzw. die Keratosis pilaris, mag man sie nun zur Ichthyosis zählen oder nicht, ferner die Pityriasis rubra pilaris (Lichen ruber acuminatus), die Akanthosis nigricans die Psorospermosis Darier 
und endlich die eingangs schon kurz gestreifte Krankheitsgruppe der Keratosis follicularis.

Ist nun unser Fall irgend einer dieser Erkrankungen zugehörig? Ich kann auf eine differentialdiagnostische Besprechung aller dieser Krankheitsbilder nicht eingehen, weil das den Rahmen dieser Arbeit weit iiberschreiten würde, und da ich doch nur, mangels hinreichender eigener Erfahrung, ein Exzerpt aus den Darstellungen der Lehr- und Handbücher unserer Disziplin geben müßte. Ich beschränke mich deshalb darauf, zu sagen, daß ich, soweit die klinischen Erscheinungen unserer Beobachtung überhaupt eine Diagnose gestatteten, auf Grund literarischen Studiums und durch die vielfachen, aus reicher Erfahrung geschöpften Belehrungen meines Chefs und Lehrers zu der Überzeugung gelangte, unseren Fall unter die Krankheitsgruppe der Keratosis follicularis einreihen zu müssen. Bevor ich jedoch diese Ansicht näher begründe, sei das Ergebnis der mikroskopischen Untersuchung mitgeteilt.

Was die Epidermis betrifft, so fällt folgendes an ihr auf: die Epidermis ist in ihrer Gesamtheit von einer sehr kompakten, außergewöhnlich breiten Hornschicht überzogen; dieselbe ist so stark ausgebildet, daß sie im Gesicht z. B. an vielen Stellen ebenso dick erscheint wie die ürbigen Teile der Epidermis $z u \cdot \cdot$ sammen, während sie am Arm zwar an sich ebenso breit, jedoch im Verbältnis zu den übrigen Schichten der Oberhaut weniger stark entwickelt ist. Im übrigen zeigt die Épidermis an Schnitten durch den Arm annähernd normale Verhältnisse: die Körnerschicht ist erhalten, das Rete malpighi nicht verbreitert, vielleicht hie und da etwas atrophisch, die interpapillären Epithelzapfen gut entwickelt. Im Gesicht dagegen erweist sich das Stratum malpighi fast uiberall als atrophisch, auf wenige Zellagen reduziert; die Papillen fehlen so gut wie vollständig, so daf die Epidermis gewissermaßen wie ein schmales, glattes Band die Cutis üherzieht. Die bei weitem auffallendsten und natürlich sofort in die Augen springenden Verhältnisse bieten jedoch die den Haaren und Talgdrüsen gemeinsamen Follikel dar. Dieselben sind nämlich ausnahmslos in ihrem obersten Abschnitt mehr oder weniger bedeutend erweitert, indes nicht immer in der gleichen Form und Art. Es resultiert entweder eine Trichter- oder Zylinderform, oder eine becherförmige, bisweilen auch mehr tulpenkelchähnliche oder kugelförmige Gestaltung dieser Teile. Was nun den Inhalt dieser Gebilde anlangt, so nimmt man wahr, wie die der Nachbarschaft des 
Follikels angehörige Hornschicht sich kontinuierlich längs der Ausbuchtung fortsetzt, und da $\beta$ das Lumen von mehr oder minder dicht gefügten Hornlamellen ausgefüllt ist. Die weitere Wandung der Follikelausbuchtung wird nun von einer zwei-, vielfach auch nur einfachen Lage meist außerordentlich langgestreckter Körnerzellen gebildet, denen weiterhin als $\mathrm{Ab}$ grenzung gegen die Cutis eine meist erheblich reduzierte, bisweilen nur noch zwei Zellreihen aufweisende Keimschicht folgt. Hie und da fehlt in einem erweiterten Follikelhalse an umschriebener Stelle die Körnerschicht auch ganz. Gegenüber diesem mächtig erweiterten obersten Follikelabschnitt erweckt nun der übrige Teil nicht selten den Eindruck eines kleinen Anhängsels, ist aber meist doch gut erhalten; bisweilen ist an der Übergangsstelle des erweiterten Follikelteiles in den übrigen Abschnitt eine fast rechtwinklige Abknickung des letzteren zu konstatieren. Was ferner die Haare betrifft, so fehlen dieselben fast stets weder in den tieferen, nicht veränderten, noch in den erweiterten Abschnitten der Follikel; jedoch sieht man nur selten das Haar in gerader Richtung mitten durch die Hornmassen ziehen, meist nimmt es vielmehr einen gewundenen Verlauf, was ohne weiteres daran zu erkennen ist, daß es in einem Schnitte mehrfach in verschiedenen Richtungen getroffen erscheint. Nur in zwei Follikeln, den kleinsten, die mir bei Durchmusterung von etwa 200 Schnitten überhaupt zu Gesicht gekommen sind, ist von Haarresten nicht das mindeste zu entdecken und doch dokumentiert die Anwesenheit von Haarbulbus und Haarpapille die Follikelnatur dieser Gebilde.

Wenden wir uns nunmehr der Reschaffenheit der Follikelöffnungen zu, so ist zu erwähnen, daß der Durchmesser derselben oft kleiner als der größte Querdurchmesser des zuzugehörigen erweiterten Follikelhalses ist, nicht selten diesen erreicht, ihn aber nur dann übertrifft, wenn eine trichterförmige Erweiterung besteht. Gerade an den Follikeln nun, die einen verhältnismäßig engen Eingang besitzen, ist es interessant zu konstatieren, wie im einzelnen Schnitt sehr häufig die Hornschicht der allgemeinen Hautdecke kontinuierlich über die Follikelöffnung hinweg zieht. Verfolgt man aber einen derartigen Follikel auf Serienschnitten weiter, so erscheint ausnahmslos an einer Stelle, die stets der stärksten Ausdehnung des Follikels entspricht, diese Decke gesprengt. Hier ragen nun keineswegs immer die den Follikel ausfüllenden Hornmassen in Form von Hornstacheln mehr oder weniger weit über die Umgebung aus der Follikelöffnung hervor, vielmehr zeigen die Hornlamellen oft eine zwiebelschalenartige Anordnung und überschreiten vollkommen geschlossen mit dem einen Pol 
die Öffnung um ein weniges. An einer Reihe von Follikeln, wohl an der Minderzahl, fehlen dieser die Offnung schließende Horndeckel oder Reste desselben vollkommen, und zwar sind das stets mit weiter Öffnung versehene. An diesen sieht man auch am schönsten die Hornstachelbildung, so daß der Eindruck erweckt wird, als bestehe die Ausfüllung des Follikels aus in einander geschachtelten Horntrichtern bez. Hornbechern.

Im Bereiche der Schweißporen ist von einer solchen Ausbuchtung, wie sie eben geschildert wurde, nichts zu bemerken, und auch an anderen Stellen der Haut vermißt man etwas derartiges vollkommen, mit Ausnahme einer einzigen, ganz oberflächlichen, Hornmassen enthaltenden Einstülpung in unmittelbarer Nachbarschaft eines Follikels.

Soviel über die Vorgänge an der Epidermis.

Die Cutis betreffend ist zunächst eine im allgemeinen mäßige, teils herdförmige, teils mehr diffuse Zellinfiltration in allen Schichten zu konstatieren, namentlich aber um die Follikel herum. Diese entzündliche Infiltration ist im Gesicht stärker ausgesprochen wie am Arm, auch zeigt ersteres die bekannten Erscheinungen der senilen Degeneration. An dem Aufbau der Zellinfiltrate nehmen vorwiegend Rund- und Bindegewebszellen teil, während Mastzellen und polymorphkernige Zellen spärlich sind und Plasmazellen ganz fehlen.

Ferner ist, entsprechend dem klinischen Bilde, ein auffallender Pigmentreichtum nachweisbar. Dieses Pigment, das keine Eisenreaktion gibt, findet sich schon sebr reichlich in den tieferen Lagen des Rete malpighi, liegt aber in besonders großen Mengen in der subepithelialen Schicht der Lederhaut. Und zwar ist es teils frei teils in unregelmäBig gestalteten, mit zahlreichen Ausläufern versehenen Zellen in Form von goldgelben oder auch mehr gelbbraunen Schollen und Körnern deponiert. Oft ist das Pigment so dicht angehäuft, daß man kaum noch Kern und Zellkonturen wahrnehmen kann. Interessant ist auch noch, daß sehr häufig das Pigment in besonders reichlichem Maße um die Follikelöffnungen' herum angehäuft erscheint.

Von Talgdrüsen ist weder in den Schnitten vom Arm noch in denen ron der Stirn auch nur die geringste Spur zu entdecken. Die Schweißdrüsenknäuel erweisen sich als mehr oder minder atrophisch.

Greifen wir nun die Hauptzüge des klinischen und dieses bistologischen Bildes unserer Beobachtung heraus, so sind das folgende: symmetrisch an Händen und Vorderarmen, im Gesicht und Nacken lokalisierte, als Follikelhyperkeratose imponierende Hautaffektion, mit auffallender, im Gesicht besonders intensiver Pigmentierung vergesellschaftet und ron starkem Juckreiz 
begleitet; die Haut im Bereiche der erkrankten Partien ungewöhnlich trocken; Entwicklung der Erkrankung etwa im Lanfe des letzten halben Jahres. Mikroskopisch: diffuse Hyperkeratose, was man makroskopisch mehr vermuten als beweisen konnte; dem klinischen Bilde entsprechend eigenartige, durch die Hyperkeratose bedingte Formveränderungen der obersten Follikelabschnitte; am Arm annähernd normaler Zustand, im Gesicht einfache Atrophie der Epidermis; Pigmentablagerung, in besonders reichlicher Menge im Gesicht, im Bereiche der tiefsten Schichten des Rete malpighi und namentlich der oberflächlichsten Lagen der Cutis; vollkommener Schwund der Talgdrüsen; im allgemeinen mäßige, im Gesicht erheblichere, entzündliche Erscheinungen in der Cutis, speziell um die Follikel herum.

Hatten wir schon auf Grund der Entwicklung und der klinischen Erscheinungen des Leidens unserer Pat. die oben erwähnten, mit Hyperkeratose und z. T. auch mit Pigmentablagerung einhergehenden Erkrankungen ausschließen zu müssen geglauht, so ließ die mikroskopische Untersuchung auch die letzten Zweifel an der Zugehörigkeit unseres Falles zur Keratosis follicularis in uns schwinden. Selbst die Möglichkeit, die wir immer im Auge behalten hatten, es könne sich hier um eine abnorme Form von Psorospermose trotz der klinischen Differenzen handeln, mußte nach dem Ergebnis der mikroskopischen Untersuchung fallen gelassen werden. Haben sich auch leider die grains und corps ronds nicht als absolut charakteristische Befunde bei der Psorospermosis Darier erwiesen, da man dieselben bei allen möglichen anderen Affektionen z. B. in Carcinomen, beim Lichen, bei Pityriasis rubra u.s.f. gefunden hat, so sind diese Gebilde doch, namentlich ihr gehäuftes Auftreten, ein hervorstechendes Kennzeichen der Dar ierschen Krankheit. Und sie fehlen in unserem Falle vollkommen. Indes davon abgesehen, wie kompliziert gestalten sich die histologischen Veränderungen bei der Psorospermose! Man höre darüber nur Jarisch: Das Rete malpighi ist gewuchert und seine Zapfen dringen tief gegen das Corium vor. Dem tieferen Eindringen der Retezapfen entspricht ein welliger Kontur der Grenzlinie zwischen Rete malpighi und 
den verdickten, mehr oder minder locker gefügten Hornschicht, indem diese sich mehr oder minder tief in die Retezapfen einsenkt und kompakte Hornzapfen bildet, wie solche auch die Follikelöffnungen erfüllen, aber ohne eine besondere Vorliebe für diese zu verraten. Dazu gesellt sich eine deutlich entwickelte Körnerschicht, eine Lückenbildung in den unteren Schichten des Rete $u$.w. m. Wie einfach und wie anders liegen demgegenüber die Verhältnisse in dem Falle unserer Beobachtung! Das ganze Bild ist beherrseht durch "die Hyperproduktion und Hyperkohäsion der Hornschichte", wie Š a mberger sich ausdrückt.

Treten wir nunmehr in eine Besprechung der Keratosis follicularis (Morrow) bzw. Keratosis follicularis contagiosa (Brooke) ein, so ist vorweg zu bemerken, daß das vorhandene Material ein außerordentlich geringes ist und z. T. kaum oder gar nicht verwertet werden kann, da es sich vielfach um mehr oder minder kurze Demonstrationen in gelebrten Gesellschaften handelt. Besonders arm an Originalarbeiten über diesen Gegenstand ist die deutsche Literatur, und zwar haben sich, soweit ich sehe, nur Unna und neuerdings Šamberger eingehend mit dieser Krankheit befaßt.

Morrow berichtet über einen 21jährigen Seemann, dessen Leiden 5 Jahre vor der ersten Untersuchung begann. M. fand eine bräunliche Verfärbung, am ausgesprochensten auf dem Abdomen, auf der Schulter und äußeren Lendenseite. Mit Ausnahme des Gesichtes, der Hohlhände und Fußsohlen war der ganze Körper von einer eigentümlichen Veränderung der Follikel befallen. Es fanden sich comedoähnliche, grauliche oder dunkle Massen, welche z. T. in Gestalt von stachelartigen Verlängerungen mehr oder weniger hervorragten. Die darüber streichende Hand empfand ein rauhes Gefühl, ähnlich dem einer Kalbszunge. Kein entzündlicher Zustand der Follikel. Die Untersuchung des Follikelinhaltes ergab einen Mangel an fettiger Materie und eine Zunahme der hornigen Elemente. Die mikroskopischẻ Untersuchung zeigte, daß die Hornschicht verdickt, das Rete unverändert war; das Corium zeigte einige erweiterte Blutgefäße. M, verlegt die Ursache der Erkrankung in die Talgdrüsen, indem es zur vorzeitigen Abstoßung der Talgdrüsenepithelien komme, womit vermutlich eine Atonie der Drüsen und eine dadurch bedingte Sekretstauung verbunden sei. Durch letztere komme es dann zur Eintrocknung und Verhärtung des Inhaites und schließlich werde der verhornte Inhalt über die Oberfläche der Haut emporgedrängt. 
B r o o ke entwickelt im internationalen Atlas seltener Hautkrankheiten folgendes Bild von seiner Keratosis follicularis contagiosa unter ausdrücklicher Betonung ihrer Identität mit dem soeben skizzierten Falle Morrows: Es bestand zunächst ein Ausschlag aus schwarzen kleinen Punkten im Nacken (21 Monate vor der Aufnahme des 6jährigen Kindes). Daraus wurden erhöhte Papeln und das ganze Gebiet schmutzig gelb. Darauf wurden die Akromien und die Außenseite der Arme befallen, wobei schließlich Papeln und braune Pjgmentierung derselben und der umgebenden Haut entstanden. Es entwickelten sich ferner comedoartige Pfröpfe und kleine stachelförmige Gebilde. Die Haut im ganzen war trocken, die kleinen Rhomboide der Oberfläche traten dentlicher hervor. Nichts gibt sagt, B., „eine bessere Idee von dem Gefühl, das die Oberfläche erzeugte, als der übliche Vergleich mit dem Überstreichen mit der Hand über eine Küchenreibe". Die größeren Papeln erschienen bisweilen fleischartig; die Krankheit, die schließlich auch Oberlippe, Stirn und Wangen erfaßte, zeigte den höchsten Grad der Entwicklung an der hinteren Achselfalte; diese Hautpartie war wie mit einem Haafen kleiner Wangen bedeckt, aus deren Oberfläche gebogene, verhornte Zapfen von 2-3 $\mathrm{mm}$ Länge hervorragten.

Sehr bemerkenswert ist, daß in einer Familie von 7 Kindern 6, in einer weiteren Familie alle 3 Kinder an dem gleichen Leiden erkrankten, während in einer dritten Familie dies nur bei einem einzigen Kinde eintrat. Infolge dieses gehäuften Auftretens glaubt B. seiner Keratosis das Beiwort contagiosa zulegen zu müssen.

Anatomisch handelt es sich nach Brooke um ein hyperplastisches Wacbsen der Epithelzellen mit einer Modifikation des Verhornungsprozesses, welche denselben eine ungewöhnlich lange Vitalitätsperiode gestatte und dauernde Adhäsion derselben zulasse. Der Hauptangriffspunkt sei der gemeinsame Follikel der Haare und Talgdrüsen, der Prozeß lokalisiere sich aber auch an den obersten Windungen vieler Schweißdrüsen und in einigen tiefer gehenden Furchen der Haut. Die Talgdrüsen seien oft intakt, und wann sie ergriffen würden, so sei das eine sekundäre Erscheinung. Schließlich betont B. die auffällige Übereinstimmung seiner Bilder mit der Darierschen Krankheit, doch sei nichts von den grains und corps ronds u. s.f. zu finden, auch fehlten papillomatöse Bildungen, sowie Geruch und Fettigkeit der Haut. Das Charakteristikum seiner Krankheit sei eine Keratose, bedingt offenbar durch „irgend einen von außen auf das Epithel einwirkenden Faktor".

Unna, welcher Gelegenheit hatte, histologische Präparate des Brookschen Falles und einen Fall von mehr lokalisierter Keratosis follicularis zu untersuchen, konnte die mikroskopischen Befunde Brookes nur bestätigen, rechnet aber die Erkrankung ibrem ganzen Charakter nach zu den oberflächlichen Entzündungen, den Hautkatarrhen, und stelit sie zur Ichthyosis und Keratosis pilaris.

Endlich teilte S a mberg e r vor kurzem folgende Krankengeschichte mit: Bei einer 25jährigen Frauensperson finden sich etwa 1 Jahr nach 
Beginn des Leidens an beiden Handrücken und auf den unteren Dritteln beider Vorderarme, und zwar auf der Volar- und Dorsalseite, eine Menge zerstreuter, isolierter, konischer Effloreszenzen, die in der Regel linsengroß sind. An ihrer Spitze sitzt eine hirsekorngroße Kruste oder eine kleine Öffnung. Die natürlichen Hautfurchen sind mehr als gewöbnlich ausgeprägt, sonst keine Veränderungen. Die Affektion hatte mit der Ent-wicklung kleiner, hirsekorngroßer, intensiv juckender Papeln begonnen.

Die histologische Untersuchung führte $\breve{S}$. zu dem Resultate, daß es sich urn eine Keratosis follicularis handele, bedingt durch Hyperproduktion and Hyperkohäsion der Hornschichte. Von dem gleichen Prozesse sei aber auch die benachbarte Hautoberfläche ergriffen, und es sei daher zweckmäßiger, die Erkrankung als Keratosis pseudofollicularis zu bezeichnen.

Von, weiteren Autoren, die über gleiche oder ähnliche Beobachtungen berichtet haben, führe ich hier, ohne auf Vollständigkeit Anspruch zu erheben, folgende an: Neely, Gr. Little, Sokoloff, Sabolitsky, Saalfeld, Halberstädter, Blaschko u. a. m. Ob alle diese Fälle hierher gehören, wage ich um so weniger zu entscheiden, als mir dieselben zu einem Teil nur aus Referaten bekannt sind; andere dagegen sind nur kurz publiziert, so daß schon aus diesem Grunde ein einigermaßen sicheres Urteil nicht erlaubt erscheint.

Nur hinsichtlich des B lasch ko schen Falles, in dem sich bei einem 53jährigen Metallschleifer am Vorderarm und Rücken beider Hände an Stelle der Haare kleine schwarze Punkte befanden und durch Druck auf einen solchen Punkt ein comedoähnlicher, aus Hornmassen bestehender Inhalt entleeren ließ, möchte ich bemerken, daß cih durchaus die Auffassung $\breve{S} a m b e r g e r s$, die übrigens in der Diskussion über den Fall bereits Behrend vertrat, teile, wonach es sich dort um Folgezustände gebandelt habe, die durch mechanische Verstopfung der Haarbälge entstanden seien. Gehört also meines Erachtens diese Beobachtung B la s c h k os nicht zur Keratosis follicularis, so bin ich anderseits in Übereinstimmung mit G a s s m a n $\mathrm{n}$ und Sa $\mathrm{m}$ b e r g e r der Meinung, daß der bekannte Lessersche Fall von Ichthyosis follicularis nicht als Icbthyosis angesehen werden darf, sondern vielmehr als der Krankheitsgruppe der Keratosis follicularis zugehörig betrachtet werden muß. Dies näher zu begründen muß ich mir an dieser Stelle versagen und verweise daher auf die diesbezüglichen Ausführungen $G$ assmanns in seinen Untersuchungen über Ichthyosis und ichthyosisähnliche Krankheiten.

Dürfen wir nunmehr, wenn wir einen Vergleich zwischen den Beobachtungen und Untersuchungen von Morrow, Brooke, Unna und Šamberger und unserem Falle ziehen, an unserer 
bereits mehrfach geäußerten Ansicht, daß unsere Beobachtung eine Keratosis follicularis ist, festhalten oder nicht? Ich glaube, diese Frage bejahen zu dürfen.

Es handelt sich nach den vorliegenden Literaturangaben um eine im Verlaufe vieler Monate and Jabre sich entwickelnde, bei Kindern scheinbar kontagiös, bei Erwachsenen sporadisch auftretende Hautkrankheit. Sitz der Affektion sind besonders die Streckseiten der Extremitäten, aber auch deren Beugeflächen, ferner Nacken, Rumpf, Gesicht und Nates. Das Leiden beginnt, häufig von starkem Jucken und in der Mehrzahl der Fälle von einer immer intensiver werdenden Pigmentierung begleitet, mit der Bildung kleiner schwarzer Punkte, aus denen allmählich erhöhte Papeln, comedoähnliche Pfröpfe und kleine, stachelförmige Gebilde werden. Die Erkrankung macht klinisch den Eindruck einer an die Follikel gebundenen Keratose, indes ist auch die Oberflächenfelderung der dazwischen liegenden Haut, die im übrigen eine auffallend trockene Beschaffenheit annimmt, deutlicher ausgesprochen als in der Norm. Die Affektion heilt in der Mehrzahl der Fälle nach einiger Zeit spontan oder unter Applikation milder bzw. keratolytischer Salben ab.

Pathologisch-anatomisch besteht stets eine Hyperkeratose, die nicht nur auf die Follikel beschränkt ist, sondern auch an den dazwischen liegenden Hautpartien in mehr oder weniger hohem Grade nachweisbar ist. Diese Hyperkeratose führt zu eigenartigen Formveränderungen der Follikel, die neben der Pigmentierung dem klinischen Bilde seine Eigenart verleiben. Das Pigment jst überwiegend in den obersten Schichten der Cutis abgelagert, aber auch in den tiefsten Schichten des Rete malpighi vermehrt. Dieses letztere ist bald unverändert, bald hyperplastisch, bisweilen auch atrophisch. Im Corium sind mehr oder minder erhebliche, im allgemeinen mäßige entzündliche Erscheinungen nachweisbar.

Diese kurze Darstellung des Krankheitsbildes, wie sie sich aus einer Zusammenfassung der oben mitgeteilten Fälle ergibt, und die klinischen Erscheinungen und mikroskopischen Befunde bei unserer Beobachtung weisen, wie wohl ohne weiteres ersichtlich ist, eine weitgehende Übereinstimmung auf. Freilich fehlt es auch nicht an Differenzen, wie ebenfalls aus einem Vergleich unserer Krankengeschichte und mikroskopischen Untersuchungsergebnisse mit den übrigen Fällen sofort hervorgeht; aber auch diese letzteren weichen unter einander teilweise nicht unerheblich ab, wie die mitgeteilten Tatsachen lehren. Am nächsten steht unser Fall zweifelsohne demjenigen Brookes. Wir sehen wie bei Brooke z. T. die gleiche Lokalisation (obere Extremitäten, Nacken, Gesicht) ; die Haut ist im Bereiche der Erkrankung intensiv pigmentiert, die 
Oberflächenfelderung deutlicher als normal ausgeprägt; wir konstatieren die Anfänge der Erkrankung in Form kleiner, schwarzer Punkte, wir sehen kleine, stachelförmige Knötchen und Papeln, den Follikeln entsprechend. Und wenn wir nun in unserem Falle eine Entwicklung von Papeln bis zu Linsengröße und darïber, wenn wir eine Konfluenz zu größeren Plaques vermissen, wenn wir vergebens nach Hautpartien suchen, die wie mit einem Haufen kleiner Warzen bedeckt erscheinen, aus denen Hornstacheln bis zu 2-3 mm Länge hervorragen so ist zu bedenken, daß diese letzteren Erscheinungen bei Personen sich zeigten, deren Leiden, unbeeinflußt durch irgend eine Behandlung, bereits über ein, mehrere, ja viele Jahre sich binzog, als sie den betreffenden Autoren zu Gesicht kamen. Bei unserer Patientin lag dagegen, als wir sie zum ersten Male sahen, der Beginn der Affektion etwa $1 / 2$ Jahr zurück. Es ist daher keineswegs unmöglich, daB sich auch in unserem Falle im Laufe der Zeit Erscheinungen obiger Art herausgebildet hätten, wären wir nicht sofort energisch eingeschritten. Daß diese Annahme nicht ganz unberechtigt ist, scheint mir der weitere Verlauf in unserem Falle zu beweisen.

Die Affektion machte nämlich, wie wir uns wiederholt äberzeugen konnten, insofern Fortschritte, als sie auf die Oberarme übergriff und auch am Nacken, freilich äußerst langsam, tiefer hinabstieg, während die ursprünglich befallenen Partien trotz energischer Anwendung keratolytischer Salben ihr Aussehen beibehielten oder dasselbe nur unbedeutend im Sinne eines Rückganges der Erscheinungen änderten. Erst seit einigen Wochen scheint sich, wenigstens nach den brieflichen Mitteilungen der Patientin, eine wesentliche Besserung geltend zu machen.

Auch in dieser Hartnäckigkeit des Leidens bei unserer Patientin zeigt sich eine Differenz gegenüber der überwiegenden Mehrzahl der bisherigen Beobachtungen. Ich glaube indes nicht, daß dieser letztere Umstand und vor allem die oben erwälnten quantitativen Abweichungen hinsichtlich der klinischen Erscheinungen dazu berechtigen, von der Diagnose Keratosis follicularis abzugehen, zumal die beiden klinischen Hauptcharaktere der Krankheit: Hyperkeratose und Pigmentierung in so ausgesprochener Weise vorhanden sind. 
Was weiter das histologische Bild betrifft, so findet sich, wie in allen bisherigen Beobachtungen, das, was dem krankhaften Prozesse unter dem Mikroskop den Stempel des Eigenartigen aufdrückt, nämlich eine diffusse Hyperkeratose, die an den Follikeln zu jenen eigenartigen, ausführlich beschriebenen Deformationen geführt hat. Es fehlt freilich in unserem Falle die Hyperplasie der Keimschicht, vielmehr ist dieselbe annähernd normal, im Gesicht sogar atrophisch. Aber diese Hyperplasie ist ja keineswegs konstant, Morrow vermißte dieselbe z. B. Dagegen konnten sie Brooke, Unna und vor allem auch Šamberger in ausgesprochenem Maße nachweisen. Und was die teilweise Atrophie des Rete malpighi in unserem Falle betrifft, so ist dieselbe wohl im Bereiche der veränderten Follikel auf den Druck der aufgestapelten Hornmassen zurück zu führen, also als Druckatrophie aufzufassen, die mehr diffuse Atrophie der Epidermis im Gesicht hingegen wohl ein Begleitsymptom der senilen Degeneration der Gesichtshaut. Diesem letzteren Prozesse ist es meiner Ansicht nach im wesentlichen auch zuzuschreiben, daß die entzündlichen Erscheinungen in unserem Falle wenigstens im Gesicht hochgradiger zu sein scheinen als in den bisher beobachteten Fällen. Endlich ist die Ablagerung sehr reichlicher Pigmentmengen, ein Symptom, das beispielsweise in dem Š a m be r g schen Falle fehlt, in Übereinstimmung mit dem klinischen Bilde auch histologisch bei unserer Patientin ohne weiteres festzustellen.

Es unterliegt nach alledem für uns keinem Zweifel, daß unser Fall eine Keratosis follicularis analog dem MorrowBrookeschen Krankheitsbilde darstellt.

Es würde nunmehr zunächst die Frage zu erörtern sein, ob die Keratosis follicularis als eine Erkrankung sui generis angesprochen werden darf. Ich habe ja bereits die Zugehörigkeit unseres Falles zu einer ganzen Reihe von Erkrankungen abgelehnt und speziell in kurzen Worten auf die Differenzen im histologischen Bilde bei der Darierschen Krankheit und Fällen unserer Art hingewiesen. Jedoch erscheint es mir nicht unzweckmäßig, noch mit einigen Zeilen wenigstens auf einige Punkte in der Klinik der Ichthyosis bzw. Keratosis pilaris einzugehen. Gassmann definiert die Ichthyosis als eine „ange- 
borene primäre Verhornungsanomalie, die mit oder ohne Verdickung der Hornschicht einhergehen kann". Die Ichthyosis wird bereits in frühester Jugend sichtbar, nimmt langsam bis zur Pubertät an Intensität zu und gelangt niemals zur Heilung. Betreffs der Lokalisation der wahren Ichthyosis ist Gassmann der Überzeugung, daß die gesamte Hautdecke von derselben betroffen wird, und zwar hauptsächlich auch deshalb, weil er den Nachweis erbringen konnte, daß klinisch als normal zu bezeichnende Hautstellen histologisch als erkrankt sich zeigten. Demgegenüber in unserem Falle Entwicklung der Krankheit im 54. Lebensjabre, an bestimmten Stellen lokalisierte, keine diffuse Erkrankung, Pigmentierung der erkrankten Partien in einer Intensität und vor allem in einer Art und Weise, wie das bei Ichthyosis nicht beobachtet wird. Nur hinsichtlich der Heilungstendenz müssen wir uns, wie oben bereits auseinander gesetzt ist, auf die Zukunft vertrösten. Die gleichen Erwägungen haben für die Keratosis pilaris Geltung, mag man dieselbe zur Ichthyosis zahlen oder ibr eine Sonderstellung einräumen. Ich kann hier auf die mikroskopischen Verhältnisse nicht eingehen, weil das viel zu weit führen würde, indes diese kurzen klinischen Notizen allein schon berechtigen, wie mir scheint, zu der Auffassung, daß die Keratosis follicularis von der Ichthyosis trotz gewisser verwandtschaftlicher Erscheinungen abseits zu stellen ist, und ich bin in voller Übereinstimmung mit $\breve{S} \mathbf{a} \mathrm{m}$ b r g e r der Ansicht, daß wenigstens nach dem heutigen Stande unseres Wissens die Keratosis follicularis als ein Leiden sui generis anzusprechen sei.

Ist nun aber die uns hier interessierende Erkrankung ein Leiden sui generis, so muß man sich natürlich auch die Frage nach der Ursache dieser Krankheit vorlegen. Mit anderen Worten, was ist das Primäre an diesem Krankheitsprozeß, und durch welches Agens wird diese primäre Veränderung ausgelöst?

Die Hauptschwierigkeit für die Beantwortung der ersten Frage liegt, wie überhaupt bei allen histologischen Untersuchungen, mag es sich um eine Erkrankung handeln, welche es sein wolle, meines Erachtens darin, daß wir immer in gewissem Sinne bereits Fertiges zur Untersuchung bekommen. 
Ein Beitrag zur Kenntnis ungewöhnlicher Keratosisformen. 209

Ein Carcinom, und mag es noch so winzig sein, ist eben doch schon ein Carcinom, und eine Papel, mag sie noch so klein sein, ist bereits eine Papel! So auch hier! Es soll an einem in voller Entwicklung begriffenen oder vielleicht sogar schon auf der Höhe seiner Entwicklung stehenden Krankheitsbilde die primäre Veränderung festgestellt werden. Es ist klar, daß man unter solchen Umständen in seinen Schlüssen über ein gewisses $\mathrm{Maß}$ von Wahrscheinlichkeit nicht hinaus kommen kann und dem subjektiven Empfinden der weiteste Spielraum gelassen ist. Es kann im vorliegenden Falle keinem Zweifel unterliegen, daB im histologischen Bilde die Intensität der am Stratum corneum sich abspielenden Vorgänge einfach dazu drängt, in ihnen das primäre des Leidens zu suchen. Und in dieser Meinung wird man dadurch bestärkt, daß man, meines Erachtens ohne den Verbältnissen Zwang anzutun, alles übrige als Folgeerscheinungen der Hyperkeratose denten kann. Halten wir zunächst einmal daran fest, daß die Hyperkeratose eine diffuse ist, also auch die Follikel ergriffen hat, soweit dieselben von Stratum corneum ausgekleidet sind! Die besonderen anatomischen Verhältnisse der letzteren, die vor allem eine $\mathrm{Ab}$ stoßung neugebildeter Hornmassen erschweren, führen nun zu einer Aufstapelung von Hornlamellen und somit zu jenen oben näher geschilderten Formveränderungen. Wie diese im einzelnen zu stande kommen, das brauche ich an dieser Stelle um so weniger zu erörtern, als Unna, und in jüngster Zeit Š a mberger diese Verhältnisse in ausführlicher und lichtroller Weise dargelegt haben, ohne freilich zu ganz übereinstimmenden Resultaten zu gelangen. Š amberger bekämpft nämlich die Ansicht Unnas, die auch bereits Brooke vertrat, daß es an einem Teil der Follikel durch Verschluß der öffnung mit fester Hornschicht zu Retentionserscheinungen und somit zur Bildung von comedoähnlichen Cysten komme. Ich muß nach den mir vorliegenden Präparaten erklären, daß ich mit Unna im wesentlichen zwei Gruppen von Veränderungen unterscheiden kann, nämlich die bereits erwähnten Retentionserscheinungen und zweitens Bildung von Hornstacheln etc. bei von vorne herein offenem Follikel. (cf. die mitgeteilten histologischen Befunde.) 
Welcher Art ist nun aber die Verhornungsanomalie? Ist die Hyperkeratose durch verminderte Abstoßung oder durch vermehrte Anbildung oder durch beides bedingt? Klinisch schuppt die Affektion überhaupt nicht, wenigstens nicht an den Armen und im Gesicht. Die Abstoßung der obersten Hornschichten ist also keinesfalls vermehrt, vielleicht sogar herabgesetzt. Und in letzterer Ansicht wird man bestärkt, wenn man unter dem Mikroskop sieht, wie fest an einander gefügt die Hornlamellen sind. Berücksichtigt man andererseits, daß in unseren Präparaten von einer Hyperplasie der Retezellen keine Rede sein kann, im Gegenteil z. T. sogar atrophische Zustände der Epidermis bestehen, und daß in Übereinstimmung damit die Zahl der Mitosen im Stratum germinativum als eine verschwindend geringe bezeichnet werden muß, so darf man daraus wohl den Schluß ziehen, daß hier eine Hyperkeratose durch vermebrte Anbildung nicht vorliegen kann. Es dürfte also im vorliegenden Falle im wesentlichen eine Retentionshyperkeratose, um einen Ausdruck Gassmanns zu gebrauchen, anzunehmen sein. Gesellt sich num aber dazu, wie z. B. in den Fällen von Brooke und Šamberger, noch eine Überproduktion von Hornsubstanz, die in der bei jenen Fällen konstatierten Hyperplasie des Rete ohne Schwierigkeit ihre Erklärung findet, so kann man sich wohl vorstellen, daß in solchen Fällen die Intensität der Erscheinungen eine größere sein wird und muß als in unserem Falle.

Auf diese Hyperkeratose kann man nun zwanglos alle weiteren konstatierten Veränderungen zurückführen. Dieselbe bedingt innerhalb des Follikels eine Verlegung der Talgdrüsenausführungsgänge und eine um so energischere Druckwirkung auf die Drüse selbst, je weiter die Ausdehnung des Follikels fortschreitet. Beide Komponenten zusammen genommen sind aber doch wohl in ihrer Wirkung ausreichend, um einen eventuell rollkommenen Schwund der Talgdrüsen herbeizuführen. Daß die vorliegende Erkrankung sonst irgend etwas mit den Talgdrüsen zu tun hätte, wie Morrow meint, der diese Drüsen als den Ausgangspunkt der ganzen Affektion ansieht, dafür habe ich ebenso wenig wie Unna und Šamberger einen Anhaltspunkt gewinnen können. Durch Druckwirkung ist auch 
die Atrophie der Epidermis im Bereiche der veränderten Follikel zu erklären, wie erheblich an diesen Stellen Druck und Zug wirken, ist ohne weiteres ersichtlich aus den Formveränderungen, welche die Zellen des Rete hier erlitten haben. Dieser Druck der Hornmassen übt ferner fortgesetzt einen mechanischen Reiz auf die Nachbarschaft aus, und diese reagiert darauf mit entzündlichen Erscheinungen. Diese letzteren werden aber noch gesteigert durch das fortwährende Kratzen, das wiederum eine Folge des beständigen, lebhaften Juckreizes ist. Diesen letzteren hinwiederum können wir vielleicht am zwanglosesten als Effekt einer konstanten, durch den Druck der Hornmassen bedingten Reizung der sensiblen Nervenendigungen- und Apparate in der Haut deuten. Eine weitere Steigerung der entzündlichen Erscheinungen ist begründet in den Altersveränderungen, die in der Haut sich abspielen, und weil nach allen vorliegenden Untersuchungen diese senilen Prozesse am stärksten im Gesicht zur Ausbildung gelangen, so ist auch verständlich, warum in unserem Falle diese entzündlichen Veränderungen ganz besonders in der Gesichtshaut mehr ausgesprochen sind als in den bisherigen Beobachtungen. Schließlich ist auch die Ablagerung reichlichen Pigmentes im Gefolge eines hyperkeratotischen Prozesses durchaus nichts ungewöhnliches, im Gegenteil ein häufiges Begleitsymptom hyperkeratotischer, entzündlicher und sonstiger Krankheitsprozesse.

Wir kommen somit, im Gegensatz zu Unna, der diese entzündlichen Veränderungen bei der Keratosis follicularis als das primäre des Leidens ansieht und diese Erkrankung daher zu den oberflächlichen Entzündungen, den Hautkatarrhen, stellt, und in Übereinstimmung mit Šamberger zu dem Ergebnis, daß das Wesen unserer Erkrankung eine erworbene Verhornungsanomalie ausmacht, daß die Hyperkeratose das primäre ist.

Fragen wir uns jetzt, welches ätiologische Moment diese Hyperkeratose zu Wege bringt, so bleibt uns nur das offene Bekenntnis übrig, daß wir zur Zeit absolut nichts darüber wissen. Die Beobachtung Brookes, daß mehrere Kinder einer Familie nach einander von dem Leiden befallen wurden, ist bisher vereinzelt geblieben. Diese Beobachtung Brockes ist an sich gewiß sehr auffällig und läßt die Meinung des Autors, daß 
bier die Wirkung eines Kontagiums vorliege, von vorneherein durchaus nicht unberechtigt erscheinen. Aber den strikten Beweis für die Richtigkeit oder Unrichtigkeit dieser Ansicht können wir heute ebenso wenig erbringen, wie seinerzeit B r o ok e selbst, da uns das fragliche Kontagium vollkommen unbekannt ist. Auf der anderen Seite muß, wie Š a mberger richtig bemerkt, durchaus nicht immer ein Kontagium die Ursache dafür sein, daß bei Menschen, welche in den gleichen Lebensverhältnissen sich befinden, die gleiche Erkrankung sich entwickelt. Zum Beweise dafür erinnert Š a mberger mit Recht an das endemische Auftreten des Kropfes in gewissen Gegenden; auch sei diesbezüglich auf das außerordentlich häufige Auftreten der Rachitis in grolien Städten gegenüber der geringeren Verbreitung auf dem platten Lande verwiesen. Die Ätiologie der Keratosis follicularis ist also vorderhand völlig in Dunkel gehüllt.

Zum Schluß noch einige Worte über die Benennung der Krankheit. Š a mberger schlägt an Stelle des bisher gebräuchlichen Namens Keratosis follicularis die Bezeichnung Keratosis pseudofollicularis vor. Ich halte diesen Vorschlag für keine Verbesserung. Zwar läßt sich Š́amberger bei der Wahl dieses Namens von dem durchaus richtigen Gedanken leiten, man müsse den diffusen Charakter der Hyperkeratose in der Namensgebung zum Ausdruck bringen. Indes jeder wird bei dem Lesen des Namens Keratosis pseudofollicularis den Eindruck gewinnen, es liege hier ein Prozeß vor, den man klinisch als follikulär gedeutet habe, während durch die mikroskopische Untersuchung das Gegenteil festgestellt sei. Das ist aber doch keineswegs der Fall; im Gegenteil die diffuse Hyperkeratose ergreift auch die Follikel und im wesentlichen die an diesen sich abspielenden Veränderungen verleihen dem Krankheitsbilde seinen eigenartigen Charakter. Man behalte daher den Namen Keratosis follicularis bei, beschränke aber seine Anwendung möglichst streng auf die wirklich hierher gehörigen Fälle. 


\section{Nachtrag.}

Nach Abschluß dieser Arbeit erschien unsere Patientin am 16. Januar 1905 in der Klinik, um sich wieder einmal zu zeigen. Wir konnten, der in der Arbeit erwähnten schriftlichen Mitteilung der Pat. entsprechend, eine sehr erbebliche Besserung konstatieren. Die Haut war im Bereiche der erkrankten Partien viel glatter, jedoch noch ebenso trocken wie früher. Die Pigmentierung der Arme war sehr erheblich, die des Gesichtes merklich zurückgegangen. Der Prozeb hatte keine bis dahin freien Hautpartien ergriffen. Die weitere Beobachtung wird lehren, ob es zu einem vollständigen Schwund der Erscheinungen kommen wird, was, wie ja ausgeführt, auch den letzten Zweifel an der. Zugehörigkeit des Falles zur Keratosis follicularis beheben würde.

\section{Literatur.}

1. Besnier-Doyon. Lehrbuch 1891. Bd. I. p. 785.

2. Blaschko, A. Keratosis follicularis. Demonstration in der Berliner dermatologischen Gesellschaft vom 6. Mai 1890. Arch. f. Derm. u. Syph. Bd. XXIII. p. 845.

3. Brooke, H. G. Keratosis follicularis contagiosa. Internationaler Atlas seltener Hautkrankheiten. H. 7. (Hier hingewiesen auf $\mathrm{Caz}$ enave, Guibert, Hardy, Leloir et Vidal.)

4. v. Eiselsberg. Die Krankheit der Schilddrüse. Deutsche Chirurgie, Lief. 38. Stuttgart 1901.

5. Gassmann. Histologische und klinische Untersuchungen über Ichthyosis und ichthyosisähnliche Krankheiten. Ergänzungsheft zum Arch. f. Dermat. u. Syph. 1904.

6. Halberstädter. Eine eigenartige Form von Keratosis follicularis (Lichen pilaris spinulosus?). Verhandlungen der Breslauer derm. Vereinigung. Arch. f. Dermat. u. Syph. Bd. LXVII. p. 133 ff.

7. H ebra, H. Hyperkeratosis striata et follicularis. Internationaler Atlas seltener Hautkrankheiten. H. 5 .

8. L esser, E. Ichthyosis follicularis. Handbuch der spez. Pathol. u. Therapie von $\mathrm{Zi}$ iemssen. 1883. Bd. XIV. 1. Hälfte. p. $477 \mathrm{ff}$.

9. Lewand ow sky, F. Über Lichen spinulosus. Arch. f. Dermat. u. Syph. Bd. LXXIII.

10. Little, G. Londoner dermat. Gesellschaft. Sitzung vom 9. Januar 1901. Ref. Monatsh. f. prakt. Dermat. Bd, XXXII. p. 20.

11. Mibelli, V. Die Ätiologie und die Varietäten der Keratosen. Monatsh. f. prakt. Dermat. Bd. XXIV. p. 345 ff. u. p. 415 ff. 
12. Morrow, Pr. A. Keratosis follicularis associated with fissurine of the tongue and Leukoplakia buccalis. Journ. of cut. and ven. diseases. September 1886. Ref. Monatsh. f. pr. Derm. Bd. VI. p. 619.

13. Neely. Keratosis follicul. mit hochgradiger Tylosis der Fußsohlen und Hohlhände. Journal of cut. and gen.-urin. dis. Vol. 6. April 1888. Ref. Monatsh. f. prakt. Dermat. Bd. VII. p. 943.

14. Neisser, A. Über einen Fall von Keratosis follic. punctata et striata. Verhandl. d. deutschen dermat. Gesellsch. IV. Kongr. 1894.

15. Saalfeld. Keratosis follicularis universalis. Berliner dermat. Gesellschaft. Sitzung vom 5. Dez. 1899. Derm. Zeitschr. Bd. VII. p. 104.

16. Sabolotsky, A. Keratosis follicularis. Venerolog.-dermatol. Gesellschaft zu Moskau. 13./25. Nov, 1898. Monatsh. f. prakt. Dermatol. Bd. XXVIII. p. 32.

17. Š a mberger. Zur Pathologie der Hyperkeratosen. I. Keratosis follicularis. Arch. f. Dermat. u. Syph. Bd. LXXXVI.

18. Sokoloff. Demonstration eines Falles von Keratosis follicul. Ven.-dermat. Ges. zu Moskau. 12./25. Okt. 1901. Monatsh. f. pr. Dermat. Bd. XXXIII. p. 593.

19. Touton. Ätiologie und Pathologie der Akne. Verhandlungen der deutschen dermat. Gesellschaft. VI. Kongreß. 1899. (Benutzt zu den einleitenden Bemerkungen über Acné cornée.)

20. Unna. Histopathologie. p. $288 \mathrm{ff}$.

21. White a) A case of Keratosis (Ichthyosis) follicularis. Journ. of cut. and gen.-urin. dis. June 1889. Ref. Monatshefte f. prakt. Dermat. Bd. IX. pag. 533. - b) Keratosis follicularis (psorospermose folliculaire végétante). A second. case. Journ. of cut. and gen.-urin, dis. 1890. Ref. Monatsh. f. prakt. Derm. Bd. XI. p. 226.

Ferner wurden benutzt die Lehrbücher von Wolff, Jarisch, Lesser, Joseph; Enzyklopädie der Haut- und Geschlechtskrankheiten; La pratique dermatologique; die Abhandlung über Hyperkeratosen von Janovsky im Handbuch der Hautkrankheiten von Mraček; Neisser$\mathrm{J}$ adassohn: Hautkrankheiten. In Ebstein-Schwalbes Handbuch.

\section{Erkiärung der Abbildungen auf Taf. IX.}

Fig. 1. Veränderungen an der Haut des Vorderarmes. Das Bild ist aus drei verschiedenen Schnitten kombiniert. Hinsichtlich der Erklärung vergleiche man den Text.

Fig. 2. Veränderungen an der Haut im Bereiche der Stirnhaargrenze. Das Bild ist ebenfalls aus drei verschiedenen Schnitten zusammengesetzt. Hinsichtlich der Erläuterung siehe den Text. 
Fig. 1.

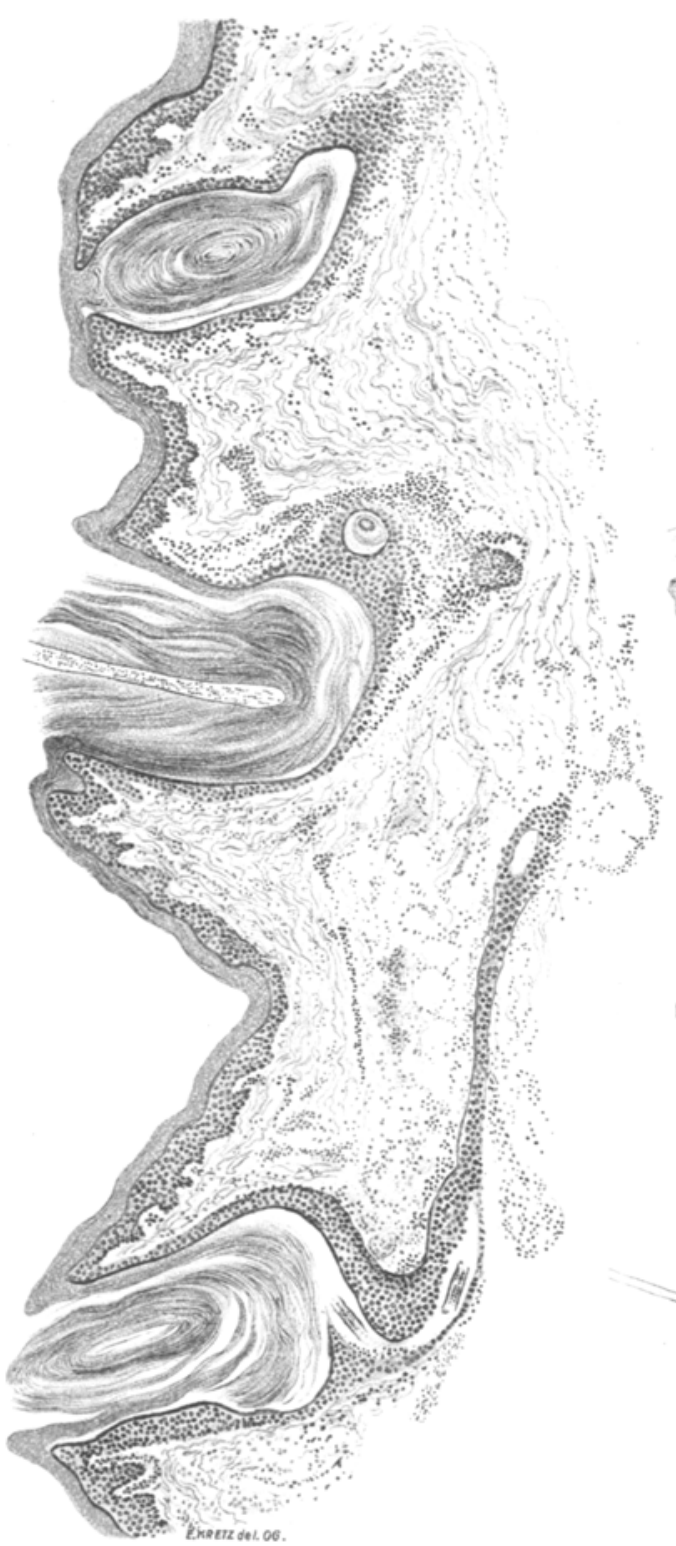

Fig. 2.

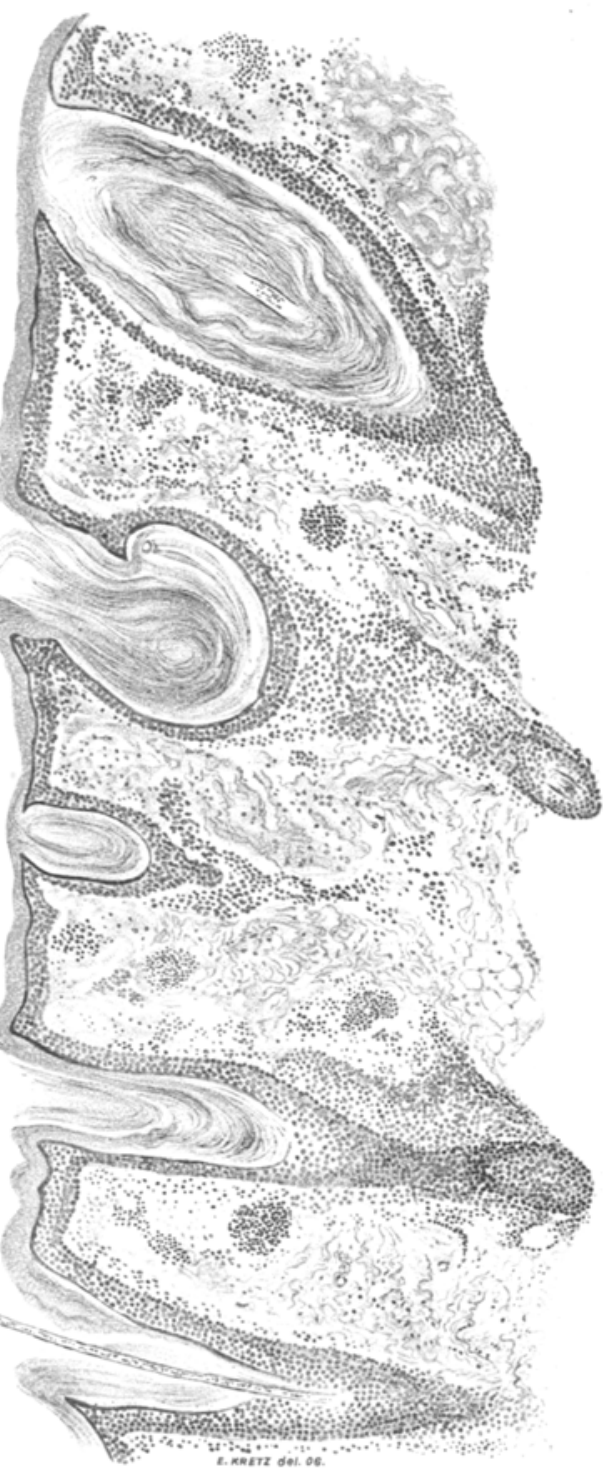

Gutmann: Zư Kenntnis ungewöhn]icher Keratosisformen. 